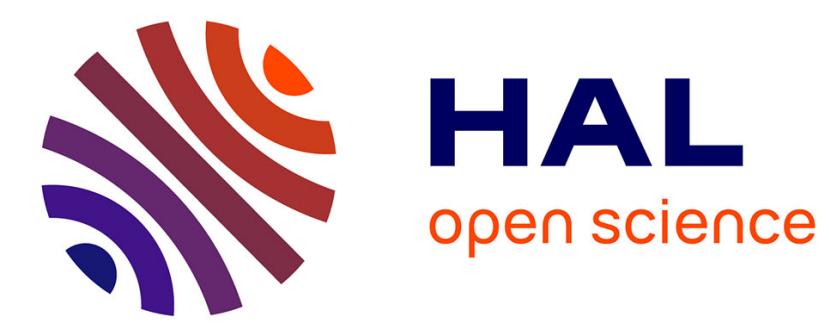

\title{
Many-particle models and short-pulse amplification in traveling wave tubes
}

Khalil Aliane, Yves Elskens, Frederic Andre, Damien F G Minenna

\section{To cite this version:}

Khalil Aliane, Yves Elskens, Frederic Andre, Damien F G Minenna. Many-particle models and shortpulse amplification in traveling wave tubes. IEEE Transactions on Electron Devices, 2021, 68 (12), pp.6476-6481. 10.1109/TED.2021.3120969 . hal-03528645

\section{HAL Id: hal-03528645 \\ https://hal.science/hal-03528645}

Submitted on 17 Jan 2022

HAL is a multi-disciplinary open access archive for the deposit and dissemination of scientific research documents, whether they are published or not. The documents may come from teaching and research institutions in France or abroad, or from public or private research centers.
L'archive ouverte pluridisciplinaire HAL, est destinée au dépôt et à la diffusion de documents scientifiques de niveau recherche, publiés ou non, émanant des établissements d'enseignement et de recherche français ou étrangers, des laboratoires publics ou privés. 


\title{
Many-particle models and short pulse amplification in traveling wave tubes
}

\author{
Khalil Aliane, Yves Elskens, Frédéric André, Member, IEEE, Damien F. G. Minenna
}

\begin{abstract}
Many-particle time domain methods are rising alternatives to particle-in-cell or frequency methods to simulate the wave-beam interactions in traveling wave tubes. We focus on two of those: our Hamiltonian discrete model DIMOHA is compared analytically against the pseudospectral method RUBEUS. Although based on two completely different approaches - the Gel'fand transform for DIMOHA and the telegraphist circuit for RUBEUS-, we surprisingly find out that they share perfectly parallel sets of equations and variables. However, we conclude that DIMOHA is more flexible than RUBEUS in terms of pitch tapering and absorbing boundary conditions. It also shows excellent stability for steady state simulation, allowing us to explain some discrepancies of RUBEUS with experimental results. These come from a standing wave pattern which is detectable in the vicinity of the sever. Finally, DIMOHA is tested for the first time with ultra-short pulses, and exhibits excellent agreement with RUBEUS.
\end{abstract}

Index terms : Time domain, Traveling wave tube, Short pulse amplification, N-body model, TWT modeling, Nonlinear wave-beam interaction.

\section{INTRODUCTION}

With the rapid development of traveling wave tube (TWT) technologies, there is growing interest in multi-frequency signal amplification, as the radiofrequency (RF) power is getting larger, and the frequency pass band wider to allow for multicarrier operation with high data flow. Thus, accurate non-linear models able to reproduce harmonic generation and intermodulation effects are required. Many such models are used to simulate the non-linear wave-beam interactions occurring in the slow wave structure (SWS) of a TWT, amongst which kinetic [1] (particle-in-cell codes) and hydrodynamic [2], [3] (eulerian and lagrangian) models, in the frequency or time domain, are the best known. In recent years however, a new class of time-domain many-particle models emerged, showing a promising balance between exhaustive physics modeling and computing time cost, as they provide faster algorithms compared to particle-in-cell codes (PIC), while being more complete than frequency domain codes.

K. Aliane is with the Centre National d'Études Spatiales, Toulouse, France (khalil.aliane@etu.univ-amu.fr).

K. Aliane and Y. Elskens (yves.elskens@univ-amu.fr) are with AixMarseille Université, CNRS, UMR 7345 PIIM, Marseille, France.

K. Aliane and F. André (frederic.andre@thalesgroup.com) are with Thales AVS, Vélizy, France.

D. F. G. . Minenna (damien.minenna@cea.fr) was with Thales AVS and is currently with CEA, DAM, DIF, F-91297 Arpajon, France.
Refs [4], [5] presented a one-dimensional (1-D) time domain method, DIMOHA, using one such many-particle description in which the wave-beam dynamics is derived in a Hamiltonian formalism. This model has been benchmarked for various industrial TWTs with mono-carrier and simple twocarrier operations. In this paper, we assess its validity for wide band multi-frequency simulations, specifically impulse amplification. Indeed, the asset of a time-domain model is that it reproduces the complex non-linear amplification of multicarrier signals in a self-consistent manner, while a frequency domain algorithm would require the injection of each individual frequency component for the same result, the extreme case being a continuous large band signal such as a pulse.

Aside from technological uses, short pulses can be used for studying TWT dispersion, gain and reflections. However, studies on this topic remain scarce [6]-[8]. Here, we study the broadband pulse amplification in the experimental TWT XWING used in previous pulses studies [6]-[8].

In prior studies, other time-domain models have been developed along similar lines to DIMOHA. In [6], - though never explicitly labeled as such -, Converse et al. developed a many-particle 1-D time-domain method, RUBEUS. A variation of Converse's model was developed by Setayesh and Abrishamian [8], extending this pseudo-spectral method to an arbitrary order of accuracy in time and space derivatives.

In addition to being many-particle 1-D time-domain methods, RUBEUS and DIMOHA bear many other similarities. In this paper, we present a thorough analytical comparison between the two models. For this comparison, we focus on

1) field decomposition and cold wave propagation,

2) wave-beam interaction modeling.

Time integration schemes will not be discussed, as we compare directly the continuous evolution equations.

\section{FIELDS DECOMPOSITION AND COLD WAVE PROPAGATION}

\section{A. RUBEUS}

To simulate the propagation of the axial component of an electromagnetic wave in the delay line of a TWT, RUBEUS is inspired by the classic telegrapher's approach, in which the slow wave structure is assimilated to a chain of transmission lines with a distributed inductance $L$ and capacitance $C$, and where the circuit parameters are related to tube properties, namely the phase velocity $v_{\varphi}$ and the coupling impedance $Z_{\mathrm{c}}$, as $L=Z_{\mathrm{c}} / v_{\varphi}$ and $C=1 /\left(v_{\varphi} Z_{\mathrm{c}}\right)$. We omit damping effects 
for this comparison. In this framework, the electromagnetic wave is propagated through the axial electric potential of the waveguide (or circuit) $V^{\mathrm{R}}(z, t)$ and an effective current $I^{\mathrm{R}}(z, t)$, where superscript $\mathrm{R}$ refers to RUBEUS.

As the Kronig-Kramers relations forbid modeling lossless frequency dependence in the time domain [9], Converse et al. incorporate dispersion into their scheme via a wavenumber $(\beta)$ dependence of the circuit parameters using a pseudospectral time-domain method (PSTD) [10]. In this scheme, the telegrapher's equations in spatial Fourier form yield

$$
\begin{aligned}
\partial_{t} \tilde{V}^{\mathrm{R}}(\beta, t) & =\frac{\mathrm{i} \beta}{C(\beta)} \tilde{I}^{\mathrm{R}}(\beta, t), \\
\partial_{t} \tilde{I}^{\mathrm{R}}(\beta, t) & =\frac{\mathrm{i} \beta}{L(\beta)} \tilde{V}^{\mathrm{R}}(\beta, t),
\end{aligned}
$$

with the Fourier transforms defined for a generic $U^{\mathrm{R}}$ as

$$
\mathcal{F}_{\beta}\left[U^{\mathrm{R}}(.)\right]=\tilde{U}^{\mathrm{R}}(\beta)=\int_{-\infty}^{\infty} U^{\mathrm{R}}(z) \mathrm{e}^{\mathrm{i} \beta z} \mathrm{~d} z
$$

so that $\mathcal{F}_{\beta}\left[\partial_{z} U^{\mathrm{R}}(z)\right]=-\mathrm{i} \beta \tilde{U}^{\mathrm{R}}(\beta)$.

As noted in [6], while this model can incorporate dispersion through a $\beta$ dependence of the circuit parameters, a simultaneous spatial dependence of those parameters is impossible. This limitation might be problematic when simulating real (e.g. tapered, i.e. with varying pitch) slow wave structures.

Considering a spatial grid mesh of step $\Delta z$, the highest spectral component that can be evaluated exactly with an FFT scheme [10] must satisfy $\left|\beta_{\max }\right|=\pi / \Delta z$. Thus we can limit the range of the integration domain in the inverse Fourier transform. Reverting to coordinate space, we obtain RUBEUS cold equations for the fields as

$$
\begin{aligned}
& \mathrm{d}_{t} V_{n}^{\mathrm{R}}(t)=\Delta z \sum_{m} S_{C}^{n-m} I_{m}^{\mathrm{R}}(t), \\
& \mathrm{d}_{t} I_{m}^{\mathrm{R}}(t)=\Delta z \sum_{n} S_{L}^{m-n} V_{n}^{\mathrm{R}}(t),
\end{aligned}
$$

where $V_{n}^{\mathrm{R}}=V^{\mathrm{R}}(z=n \Delta z)$ and the equivalent for $I_{m}^{\mathrm{R}}$, and where the propagators $S_{C}$ and $S_{L}$ are given by the Fourier transforms of $\frac{i \beta}{C(\beta)}$ and $\frac{i \beta}{L(\beta)}$ (Fig. 1 .

\section{B. DIMOHA}

A more thorough description of DIMOHA is given in Refs [4], [5]. In this model, the field representation relies on the Kuznetsov discrete model used to reduce the field degrees of freedom (DOFs) [11], and we express the RF electric and magnetic fields in a periodic waveguide as

$$
\begin{aligned}
& \mathbf{E}(\mathbf{r}, t)=\frac{1}{2 \pi} \int_{-\pi}^{\pi} V_{\beta}^{\mathrm{D}}(t) \mathbf{E}_{\beta}^{\mathrm{D}}(\mathbf{r}) \mathrm{d}(\beta d), \\
& \mathbf{H}(\mathbf{r}, t)=\frac{\mathrm{i}}{2 \pi} \int_{-\pi}^{\pi} I_{\beta}^{\mathrm{D}}(t) \mathbf{H}_{\beta}^{\mathrm{D}}(\mathbf{r}) \mathrm{d}(\beta d),
\end{aligned}
$$

where $V_{\beta}^{\mathrm{D}}$ and $I_{\beta}^{\mathrm{D}}$ are time-dependent amplitudes, factor $\mathrm{i}$ results from a technical convention, $d$ is the period (e.g. the pitch for a helix). The eigenfields $\mathbf{E}_{\beta}^{\mathrm{D}}, \mathbf{H}_{\beta}^{\mathrm{D}}$ are solutions to the Helmholtz equations, they depend only on the SWS geometry and contain dispersion and coupling properties. The longitudinal component of $\mathbf{E}_{\beta}^{\mathrm{D}}(\mathbf{r})$ on the axis for helix SWS is given [5] for $\beta>0$ by

$$
E_{\beta}^{\mathrm{D}}(z)=\beta \sqrt{\frac{v_{\mathrm{g}} Z_{\mathrm{c}} \omega_{\beta}}{d}} \mathrm{e}^{-\mathrm{i} \beta z}
$$

with the group velocity $v_{\mathrm{g}}=\partial_{\beta} \omega$, while $\mathbf{E}_{-\beta}^{\mathrm{D}}=\mathbf{E}_{\beta}^{\mathrm{D}}$.

Despite the notation, DIMOHA's time-dependent amplitude $V^{\mathrm{D}}$ does not directly represent the on-axis electric potential, contrary to the RUBEUS field variable $V^{\mathrm{R}}$ (and both $I^{\mathrm{D}}$ and $I^{\mathrm{R}}$ are shorthand variables for RF fields rather than physical charge currents). Using eq. (4), the source-free Maxwell equations give the propagation scheme for DIMOHA

$$
\begin{aligned}
\partial_{t} V_{\beta}^{\mathrm{D}}(t) & =-\omega_{\beta} I_{\beta}^{\mathrm{D}}(t), \\
\partial_{t} I_{\beta}^{\mathrm{D}}(t) & =\omega_{\beta} V_{\beta}^{\mathrm{D}}(t) .
\end{aligned}
$$

The first advantage of DIMOHA appears, as the propagation of the two variables presents a strong symmetry (which translates to a good balance in the numerical approach). Furthermore, whereas the kernel i $\beta / L(\beta)$ (Fig. 1 (c)) in RUBEUs eq. 1) diverges near $\beta \Delta z=\pi$ as the interaction impedance $Z_{\mathrm{c}}$ rapidly decays, $\omega_{\beta}$ (Fig. 11 (a)) varies moderately at band edges for typical TWTs. The field evolution equations in space representation read

$$
\begin{aligned}
\mathrm{d}_{t} V_{n}^{\mathrm{D}}(t) & =-\sum_{m} \Omega_{n-m} I_{m}^{\mathrm{D}}(t), \\
\mathrm{d}_{t} I_{n}^{\mathrm{D}}(t) & =\sum_{m} \Omega_{n-m} V_{m}^{\mathrm{D}}(t) .
\end{aligned}
$$

with $\left(V_{n}^{\mathrm{D}}, I_{n}^{\mathrm{D}}\right)$ the inverse Gel'fand transforms of $\left(V_{\beta}^{\mathrm{D}}, I_{\beta}^{\mathrm{D}}\right)$,

$$
\left(V_{n}^{\mathrm{D}}, I_{n}^{\mathrm{D}}\right)=\frac{1}{2 \pi} \int_{-\pi}^{\pi}\left(V_{\beta}^{\mathrm{D}}, I_{\beta}^{\mathrm{D}}\right) \mathrm{e}^{-\mathrm{i} \beta n d} \mathrm{~d}(\beta d) .
$$

Though very similar, the Gel'fand transform is distinct from the regular Fourier transform. Thus, we will refer to DIMOHA discrete space points $(n)$ as cells, rather than a mesh to avoid confusion with RUBEUS Fourier representations (refer to [4], [5] for details on the Gel'fand transform).

Similarly to the RUBEUS equations, the dynamics eqs (7) correspond to a chain of harmonic oscillators with coupling coefficients $\Omega_{n-m}=\frac{1}{2 \pi} \int_{-\pi}^{\pi} \omega_{\beta} \mathrm{e}^{-\mathrm{i} \beta(n-m) d} \mathrm{~d}(\beta d)$. Again, thanks to our choice of field variables, $\Omega_{n-m}$ (Fig. 1 (d)) is less jagged than $S_{L}^{n-m}$ (Fig. 1 (f)) and decays as $(n-m)^{-2}$ for moderately dispersive delay lines. This conveys another advantage for DIMOHA, as the decay of $\Omega_{n-m}$ as one moves away from the $n^{\text {th }}$ cell is faster than RUBEUS $S_{L}^{n-m}$ decay, which allows us to truncate the coupling range $N_{\text {ph }}$ down to a few cells, whereas RUBEUS would require a wider coupling range. Though the DIMOHA decomposition was constructed for ideal periodic structures, it remains a good approximation for tapered TWTs as long as the pitch and radius shifts are not too steep. Indeed, one can include tapers and defects by slightly altering parameters from cell to cell, with different dispersion and interaction properties $\left(\omega_{\beta}, Z_{c}\right)$ for each cell. This has been done in [4], where DIMOHA was successfully tested with a tapered industrial TWT, which provides it a massive advantage compared to RUBEUS. 
(a)

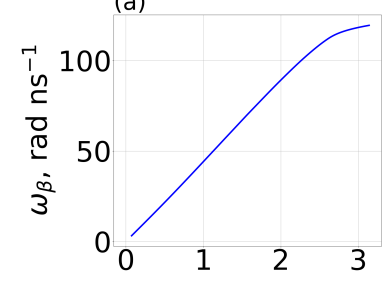

(b)
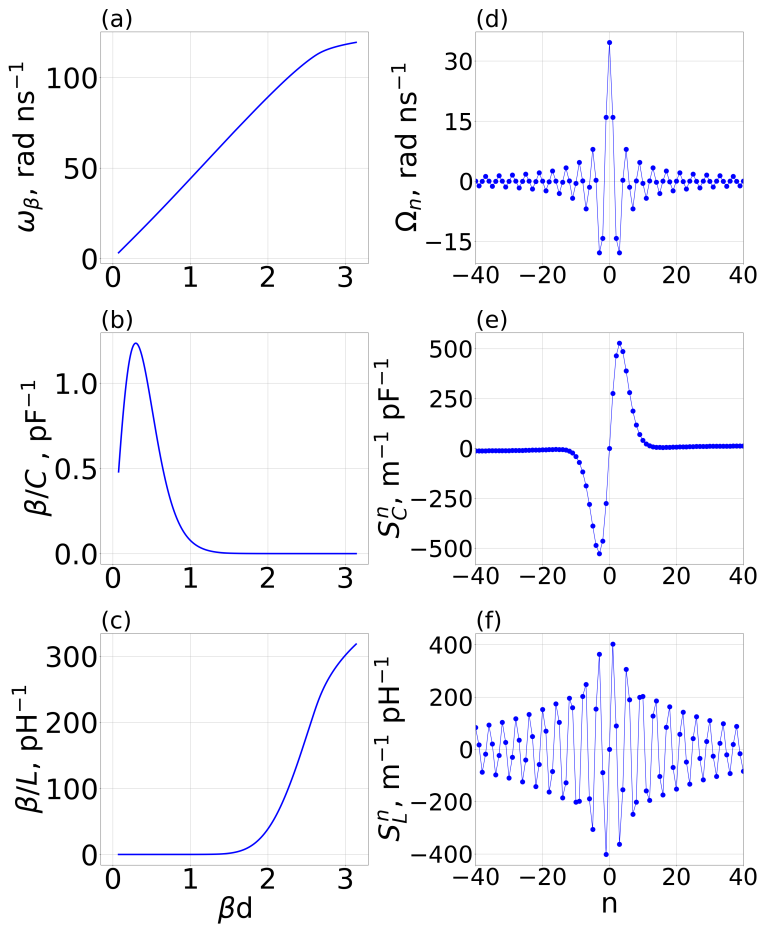

Fig. 1. Propagation kernels for the XWING TWT in both space and cell/grid representation. DIMOHA kernel $\left(\boldsymbol{\Omega}_{\boldsymbol{\beta}}, \boldsymbol{\Omega}_{\boldsymbol{n}}\right)$ (a,d), and RUBEUS kernels $\left(\frac{\beta}{C(\boldsymbol{\beta})}, S_{C}^{n}\right)(\mathrm{b}, \mathrm{e})$ and $\left(\frac{\boldsymbol{\beta}}{L(\boldsymbol{\beta})}, S_{L}^{n}\right)(\mathrm{c}, \mathrm{f})$.

\section{Connection between the models}

In both models, electrons are moved by the influence of the RF field. So, we must link the field variables $\left(V^{\mathrm{R}}, I^{\mathrm{R}}\right)$ and $\left(V^{\mathrm{D}}, I^{\mathrm{D}}\right)$ to each other as well as to physical fields. Consistency in the linear regime for space-Fourier dependence implies that the $\beta$-component of a variable does not relate to a different component $\beta^{\prime}$ of other variables, thusly

$$
\begin{aligned}
V_{\beta}^{\mathrm{D}} & =g_{\beta}^{V} \tilde{V}^{\mathrm{R}}(\beta)+k_{\beta}^{I} \tilde{I}^{\mathrm{R}}(\beta), \\
I_{\beta}^{\mathrm{D}} & =k_{\beta}^{V} \tilde{V}^{\mathrm{R}}(\beta)+g_{\beta}^{I} \tilde{I}^{\mathrm{R}}(\beta) .
\end{aligned}
$$

Substituting eqs (9) into the field evolution eqs (1) and (6), we obtain $g_{\beta}^{I}=-\mathrm{i} g_{\beta}^{V} Z_{\mathrm{c}}$ and $k_{\beta}^{V}=-\mathrm{i} k_{\beta}^{I} / Z_{\mathrm{c}}$. The values of $g_{\beta}^{V}$ and $k_{\beta}^{I}$ can then be derived by considering the physical electric field. For RUBEUS, $V^{\mathrm{R}}(z, t)$ is the physical potential from which the on-axis electric field is derived, so that

$$
E_{z}(z, t)=-\partial_{z} V^{\mathrm{R}}=\frac{1}{2 \pi} \int_{-\infty}^{\infty} \mathrm{i} \beta \tilde{V}^{\mathrm{R}}(\beta, t) \mathrm{e}^{-\mathrm{i} \beta z} \mathrm{~d} \beta .
$$

For DIMOHA, the electric field is reconstructed from the time amplitudes $V_{\beta}^{\mathrm{D}}$ and eigenfunctions $\mathbf{E}_{\beta}^{\mathrm{D}}$ through eq. 4.

To compare the two representations of the physical electric fields, one must specify the integration range for eq. (10). For simplicity, we choose a grid mesh $\Delta z$ equal to the pitch $d$ of the SWS. The equivalence of eqs (4a) and (10) requires

$$
g_{\beta}^{V}=\mathrm{i} \beta /\left(E_{\beta}^{\mathrm{D}}(0) d\right), \quad k_{\beta}^{I}=0,
$$

where DIMOHA's on-axis eigenfunction $E_{\beta}^{\mathrm{D}}(z)$ is given in eq. (5) and the contribution of $\tilde{V}^{\mathrm{R}}(\beta)$ must be negligible for $|\beta d|>\pi$. Substituting eq. (11) into eq. (9) yields

$$
\begin{array}{r}
V_{\beta}^{\mathrm{D}}=g_{\beta}^{V} \tilde{V}^{\mathrm{R}}(\beta), \\
I_{\beta}^{\mathrm{D}}=-\mathrm{i} g_{\beta}^{V} Z_{\mathrm{c}} \tilde{I}^{\mathrm{R}}(\beta) .
\end{array}
$$

Similarly, one can also map the field variables in space, using the Fourier transform of the kernels $g_{\beta}^{V}$ and $g_{\beta}^{V}$.

\section{WAVE-BEAM INTERACTION MODEL}

Now that we can switch between RUBEUS and DIMOHA field variables, the modeling of wave-beam interaction in both models can be compared. The acceleration of an electron at $z=q_{r}$ is $-\eta E_{z}\left(q_{r}\right)$ with $\eta$ the absolute value of the chargeto-mass ratio and $E_{z}$ the electric field. As we required the on-axis electric field to be the same in both models, they generate the same motion for the electrons, provided that the electrons generate equivalent source terms. Thus, we focus here exclusively on the effect of the beam on the RF field, and we omit space charge effects.

In DIMOHA [5], the effects of the beam on the wave are incorporated into eq. (6) through the current density term,

$$
\partial_{t} V_{\beta}^{\mathrm{D}}(t)=-\omega_{\beta} I_{\beta}^{\mathrm{D}}(t)-\mathrm{i} Q \sum_{r} \dot{q}_{r}(t) A_{\beta}^{\mathrm{D}^{*}}\left(q_{r}(t)\right),
$$

where $q_{r}$ and $\dot{q}_{r}$ are the space coordinate and the velocity of the macro-electron labeled $r$ along the $z$-axis, $Q$ is the macro-electron charge, and $A_{\beta}^{\mathrm{D}}(z)$ is the $\beta$-component of the vector potential eigenfunction at $z$. The smooth nature of this eigenfunction makes it possible to gather adjacent particles into macro-electrons, since their coupling is approximately the same, i.e. $A_{\beta}^{\mathrm{D}}(z) \approx A_{\beta}^{\mathrm{D}}(z+\varepsilon)$, further reducing the DOFs. This function (Fig. 2) is given [5] by

$$
A_{\beta}^{\mathrm{D}}(z)=\mathrm{i} \frac{E_{\beta}^{\mathrm{D}}(z)}{\omega_{\beta}}=-A_{-\beta}^{\mathrm{D}}{ }^{*}(z) .
$$
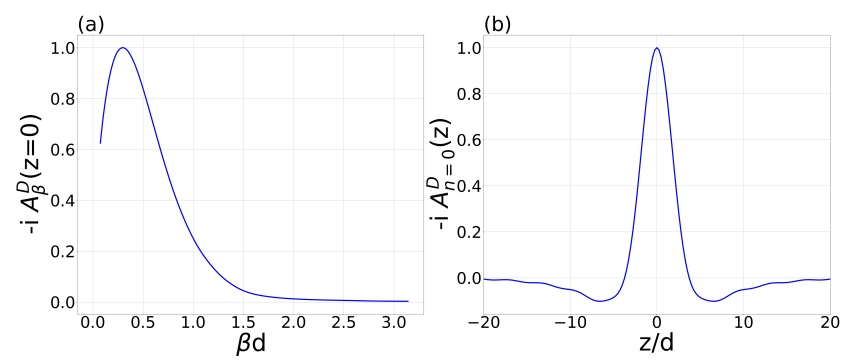

Fig. 2. Normalised amplitude $-\mathbf{i} \boldsymbol{A}_{\boldsymbol{\beta}}^{\mathrm{D}}(\boldsymbol{z}=\mathbf{0})$ of the vector potential eigenfunction (left) and shape function $-\mathbf{i} A_{n=0}^{\mathrm{D}}(\boldsymbol{z})$ (right) for XWING.

In RUBEUS, electrons are also conglomerated into finite size macro-particles, and their effect on the RF fields is taken into account by adding an image current $I^{\mathrm{b}, \mathrm{R}}$ to the modulated current $I^{\mathrm{R}}$ according to Ramo's theorem [12]. While the motion of macro-electrons is continuous, the fields are only defined on a grid. Therefore, to estimate the contribution of a macro-electron with coordinate $q_{r}$ and velocity $\dot{q}_{r}$ to the effective current $I^{\mathrm{b}, \mathrm{R}}$ at a given point on the grid, Converse et al. equip each macro-electron with a shape function 
$S^{\mathrm{R}}\left(z, q_{r}\right)=S^{\mathrm{R}}\left(0, q_{r}-z\right)$ defining the spread of the charge in space. Indeed, if electrons were treated as point particles and sampled upon their passage through grid points, this would induce noise in the grid current. The beam-induced current $I^{\mathrm{b}, \mathrm{R}}(z=n \Delta z)=I_{n}^{\mathrm{b}, \mathrm{R}}$ at grid point $n$ then reads

$$
I_{n}^{\mathrm{b}, \mathrm{R}}(t)=\frac{Q}{\Delta z} \sum_{r} \dot{q}_{r}(t) S^{\mathrm{R}}\left(n \Delta z, q_{r}\right) .
$$

In DIMOHA, electrons do not need to be given a shape of their own in regards to their effects on field variables. They can be treated as point particles, and their position is sampled over a spatial grid $z_{i}$ superposed with the field cells grid $n$. The contribution of an electron passing through a given position $z_{i}$ to the RF field coupling at a given cell $n$ is weighted by the vector potential shape function $A_{n}^{\mathrm{D}}\left(z_{i}\right)$. From a numerical standpoint, this function plays the same role as particle shape functions in RUBEUS.

As the specific choice of shape function $S^{\mathrm{R}}$ used by Converse et al. [9] - a quadratic spline function - is not essential to our comparison, we leave it unspecified. To keep our comparison in the $\beta$-representation, we use $\tilde{S}^{\mathrm{R}}\left(\beta, q_{r}\right)=$ $\mathcal{F}_{\beta}\left[S^{\mathrm{R}}\left(\cdot, q_{r}\right)\right]$. Introducing the beam effective image current $\tilde{I}^{\mathrm{b}, \mathrm{R}}(\beta, t)$ into the field evolution eqs. $(1)$ yields

$$
\partial_{t} \tilde{V}^{\mathrm{R}}(\beta, t)=\frac{\mathrm{i} \beta}{C(\beta)}\left(\tilde{I}^{\mathrm{R}}(\beta, t)+\frac{Q}{\Delta z} \sum_{r} \dot{q}_{r}(t) \tilde{S}^{\mathrm{R}}\left(\beta, q_{r}\right)\right),
$$

which the mapping eq. (12) rewrites with DIMOHA variables,

$$
\partial_{t} V_{\beta}^{\mathrm{D}}(t)=-\omega_{\beta} I_{\beta}^{\mathrm{D}}(t)+\frac{\mathrm{i} Q \beta g_{\beta}^{V}}{C(\beta) \Delta z} \sum_{r} \dot{q}_{r}(t) \tilde{S}^{\mathrm{R}}\left(\beta, q_{r}(t)\right) .
$$

Comparing eqs 17, and 13 , it appears that $g_{\beta}^{V} \tilde{S}^{\mathrm{R}}(\beta, z)$ plays a role similar to $A_{\beta}^{\mathrm{D}}{ }^{*}(z)$. One might stop the comparison here, as the coupling of beam particles to the RF field variables is estimated differently in RUBEUS and DIMOHA. However, it is interesting to look for a specific shape function $\tilde{S}^{\mathrm{R}}\left(\beta, q_{r}\right)$ that ensures the same couplings in both models, i.e.

$$
\frac{1}{d} \frac{\beta}{C(\beta)} g_{\beta}^{V} \tilde{S}^{\mathrm{R}}\left(\beta, q_{r}\right)=-A_{\beta}^{\mathrm{D}^{*}}\left(q_{r}\right),
$$

considering a space mesh $\Delta z=d$ for RUBEUS. Substituting eq. 11 for $g_{\beta}^{V}$ and eq. 14 for $A_{\beta}^{\mathrm{D}}$, we derive

$$
\tilde{S}^{\mathrm{R}}\left(\beta, q_{r}\right)=d \frac{v_{\mathrm{g}}(\beta)}{v_{\varphi}(\beta)} \mathrm{e}^{\mathrm{i} \beta q_{r}} .
$$

Thus, for a dispersionless TWT (i.e. $v_{\mathrm{g}}=v_{\varphi}$ ), the shape function would essentially be a Dirac distribution. In a sense, the TWT dispersion defines a kind of intrinsic shape function for electrons, preventing them from appearing as point-like sources in our dynamics.

In conclusion, the most striking differences between the two models might be that, whereas the electrons shape functions used in RUBEUS to weight the beam effects on the discretised electric potential are a somewhat arbitrary or at least flexible numerical tool primarily used to reduce current noise, the field eigenfunctions used in DIMOHA are directly derived from the physical vector potential acting on beam particles, and thus the coupling in DIMOHA is more physically grounded.
Furthermore, $A^{\mathrm{D}}$ shares with wavelets the properties that $A_{\beta}^{\mathrm{D}}$ is localised in $\beta$-space and $A_{n}^{\mathrm{D}}(z)$ in $z$-space, along with orthogonality properties inherited from the eigenfunctions $\mathbf{E}_{\beta}^{\mathrm{D}}$. The particular field decomposition of DIMOHA, coupled with the locality of the field functions $A_{n}^{\mathrm{D}}(z)$ (Fig. 2 and $E_{n}^{\mathrm{D}}(z)$, gives it an advantageous flexibility so as to successfully simulate tapered and defective delay lines.

\section{COMPARISON WITH EXPERIMENTS}

DIMOHA has proven to be a good candidate for TWT simulations, as its validity has already been assessed for single carrier operation on several industrial TWTs [4], [13], for two-carrier driven operation [4] and for meter-long TWTs operating in strong non-linear regimes, used to study beamplasma instabilities [5].

However, it has not yet been used to simulate multifrequency signals with arbitrary form. Assessing this capacity is important for telecommunication signals simulation. Here, we study the propagation and amplification of very short pulses in DIMOHA in an ultra-wide band experimental TWT [6]. Due to their short range, ultra short pulses are mainly present in radar technology, and their use in TWT amplification remains rather limited.

Nonetheless, short Gaussian pulse studies in TWTs are interesting, even if only for characterizing a tube. Indeed, one can derive several features such as small signal gain and phase shift over a broad range of frequencies in a single run using a Gaussian pulse. Moreover, it can be a useful tool to probe complex non-linear mechanisms involving several harmonics of multiple frequencies, and is well suited to simulate the amplification of phase coded telecommunications signals.

Finally, short pulse amplification is a good way to corroborate DIMOHA's validity for multi-frequency arbitrary form signals, as such impulses were studied by Converse $e$ t al. using XWING to assess RUBEUS. To further cement our results, we compare them to two other codes, namely MVTRAD, which is a 2.5-D self-consistent algorithm in the frequency domain conceived by Thales [14], and LATTE, another frequency domain algorithm, based on a lagrangian beam description [2]. Due to their good agreement with RUBEUS and for the sake of figures clarity, we did not add data from PaWaIC [8].

\section{A. Mono-frequency XWING characterisation}

The eXperimental WIsconsin Northrop Grumman TWT (XWING) is a helical non-tapered tube customised for research purposes [15]. It contains a sever to supress reflection and covers a broad amplification band of 2-6 GHz. Multiple sensors placed along the delay line and coupled capacitively to the circuit enable the probing of the RF field spatial evolution.

For DIMOHA simulations, we used the dispersion characteristics (Fig. 11), nominal beam parameters (cathode potential $2700 \mathrm{~V}$, current $0.2 \mathrm{~A}$ ) provided in [9], and a coupling range $N_{\mathrm{ph}}=40$. We include the sever by adding a damping term in the field eq (7)

$$
\mathrm{d}_{t} V_{n}^{\mathrm{D}}(t)=-\sum_{m} \Omega_{n-m} I_{m}^{\mathrm{D}}(t)-\alpha_{n} V_{n}^{\mathrm{D}}(t),
$$


where we choose $\alpha_{n}=\alpha \cos ^{2}\left[\left(n d-z_{\text {sever }}\right) \pi / L_{\text {sever }}\right]$ with $\alpha$ the maximum amplitude of the attenuation, $z_{\text {sever }}$ the position of the sever and $L_{\text {sever }}$ its spatial range. These parameters are adjusted to fit the attenuation profile given in [15].

A similar method is used to add perfectly matched layers (PML) at both ends of the SWS, to absorb waves exiting the tube and avoid reflections. Indeed, one of DIMOHA's assets is that it supports both forward and backward wave propagation, allowing one to simulate defects and reflections. The drawback is that an imperfect absorbing boundary condition will generate reflections in our signal, as in Fig. 3 where the RF power simulated by DIMOHA (dashed blue line) presents a standing wave pattern in steady state. The oscillations look stronger near the sever, where the backward wave is of the same order of magnitude as the attenuated direct wave. This is not a major issue, as one can prevent reflections by simulating transient states (plain blue line in Fig. 3) as in [9].

The standing wave pattern observed with DIMOHA is consistent with the measured RF power (black dots in Fig. 3), which suggests that the discrepancy between the measurements and simulations at the sever might result from reflections in the traveling wave tube. Nevertheless, a complete quantitative analysis requires further experimental studies, and is outwith the scope of this paper.

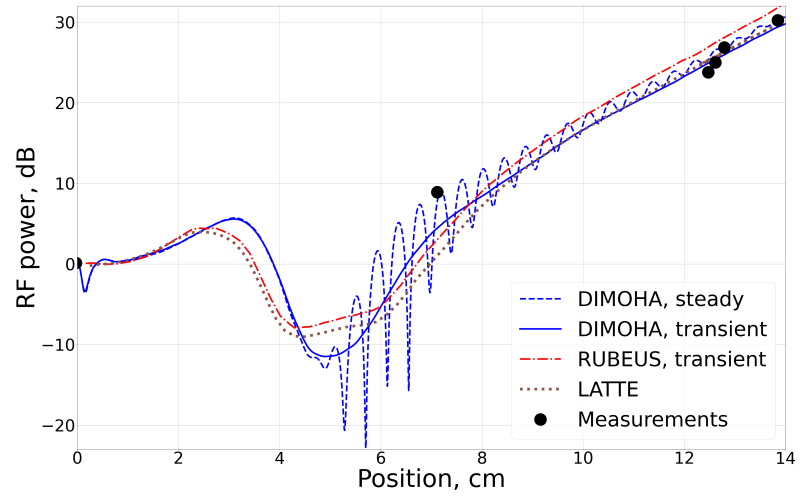

Fig. 3. RF power amplification along the SWS versus position for $f=$ $3 \mathrm{GHz}$. DIмона simulations transient (plain blue line) and steady state (dashed blue line), LATTE simulation (brown dots), RUBEus transient simulation (red dash-dot line), and measurements (black dots).
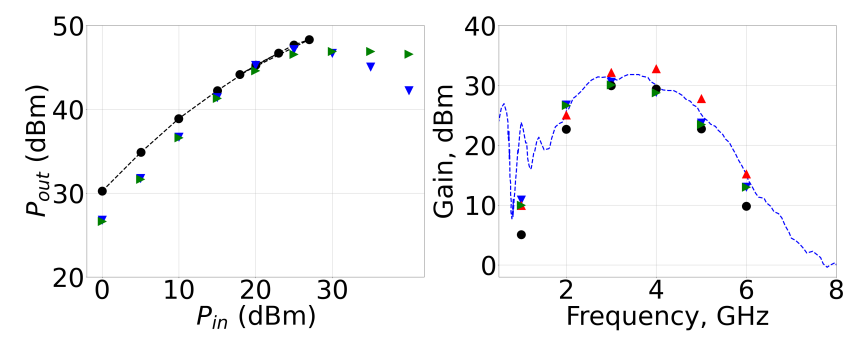

Fig. 4. Left panel : AM-AM curve for $f=4 \mathrm{GHz}$, right panel small signal gain. DIMOHA mono-carrier simulations (blue $\mathbf{v}$ ) and pulse simulation (dashed blue line), MVTRAD simulations (green $\bullet$ ), RUBEUS simulations (red $\mathbf{\Lambda}$ ) and measurements (black dots).

With the sever adjusted, we performed a mono-frequency characterisation of XWING, simulating the amplification in the gain band ranging from 2 to $6 \mathrm{GHz}$ with a frequency step of $1 \mathrm{GHz}$, each time for several input powers, looking for the saturation point at each frequency. The AM-AM curves thusly obtained are compared to those simulated with MVTRAD and with measurements [15]. Each point of the left panel in Fig. 4 took five minutes on 16 processors with DIMOHA.

The small signal gain obtained with DIMOHA is compared with experimental results and other simulations in Fig. 4 MVTRAD and DIMOHA are in good agreement, while they both differ from RUBEus results by up to $2 \mathrm{~dB}$. This is not quite significant, as the coupling impedance is read from a curve given in [9] in logarithmic scale, with a limited accuracy.

\section{B. Short impulse amplification}

In time-domain models, intermodulation and non-linear effects are taken into account self-consistently when simulating a pulse, while frequency models require the inclusion of all significant frequencies to reproduce such effects, as a finite pulse in time involves a continuum of frequencies. To assess the modeling of pulse amplification with DIMOHA, the monofrequency gain presented in section IV-A is compared to the gain obtained using the Fourier spectrum of a short $0.1 \mathrm{~ns}$ small pulse. Note that to obtain the gain in DIMOHA, one need not use the physical electric field $E(z, t)$, as we can compute it directly from the input and output values of field variables $\left(I_{n_{\text {out }}}^{\mathrm{D}}, I_{n_{\text {in }}}^{\mathrm{D}}\right)$ or $\left(V_{n_{\text {out }}}^{\mathrm{D}}, V_{n_{\text {in }}}^{\mathrm{D}}\right)$.

Negative and positive pulses were simulated in both small and large signal regimes. The results are compared to measurements and with RUBEUS simulations in Fig. 5 Note that we compare the potential amplitude measured and simulated by RUBEUS [6] to the scaled amplitude of DIMOHA's $I_{n_{\text {out }}}^{\mathrm{D}}$.

DIMOHA results for small pulses present a good agreement with measurements and RUBEUs. Agreement with PaWaIC [8] follows from the agreement of the latter with RUBEUS. In the large signals regime however, DIMOHA shows a good agreement with RUBEUS, while they both diverge from the measurements. Converse $e t$ al. suggested that this discrepancy after saturation might be due to 3-dimensional effects, as the beam radius might expand significantly in this regime where the beam bunching is stronger.

In Fig. 6, we display the signal amplitude simulated by DIMOHA in both time and space. Along the diagonal, we see the pulse propagating along the TWT, where it is attenuated in the sever $(z \sim 100 \mathrm{~d} z)$ and then amplified until the TWT exit. A portion of the signal is reflected on the sever and propagates backward in the region $(400 \lesssim t / \mathrm{d} t \lesssim 1500,0 \lesssim z / \mathrm{d} z \lesssim 80$ ), as was also observed in [8]. Another reflection occurs at the end of the TWT ( $t \gtrsim 1500 \mathrm{~d} t, z \gtrsim 200 \mathrm{~d} z$ ). The ability of DIMOHA to support backward waves makes it a helpful tool when simulating reflective defects.

\section{CONCLUSION AND PERSPECTIVES}

While time-domain self-consistent simulations are appropriate to model the complex multi-frequency wave-beam interactions in a TWT, their benefits can quickly be outweighed by their time cost, considerably larger than frequency-domain simulations. Using an order reduction model, the Kuznetsov 


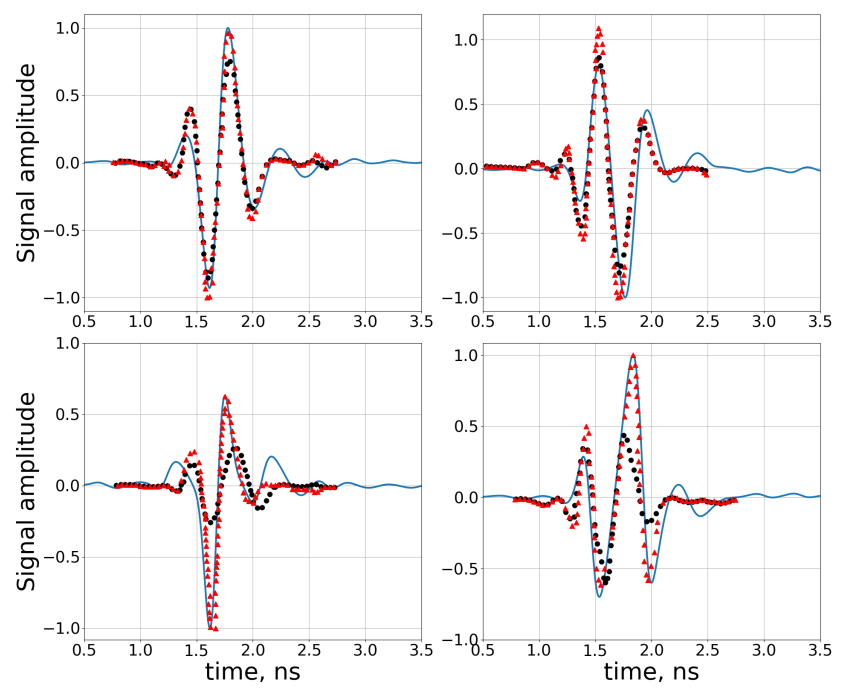

Fig. 5. Amplified time signal at the TWT exit. Left column : negative input, right column : positive input, top row : small signal regime, bottom row : large signal regime. DIMOHA simulation (blue line), RUBEUS (red $\Delta)$, and measurements (black dots).
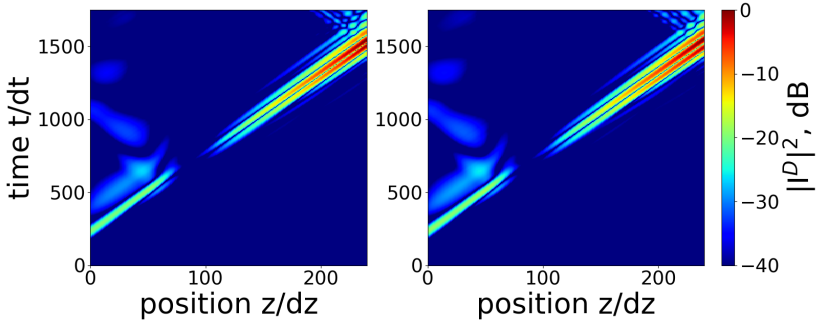

Fig. 6. Normalised signal amplitude (squared) in time and space with a positive input. (left) Small signal, (right) large signal. $\mathrm{d} z=0.58 \mathrm{~mm}$, $\mathrm{d} t=4 \mathrm{ps}$.

discrete decomposition, we developed such a time-domain algorithm, DIMOHA, with a reduced time cost.

In this paper, we compare DIMOHA analytically and numerically with a similar time-domain many-particle model, RUBEUS. We show that the wave-beam coupling in DIMOHA is assured by spatially local functions derived naturally from the dynamics, rather than relying on arbitrary particle shape functions. Moreover, the smoothness of DIMOHA's field shape function allows for effective particles conglomeration into larger macro-particles, while its spatial locality enables us to truncate the coupling range, further reducing simulation time cost, and allowing for more flexibility with the spatial structure of the waveguide. Thus, DIMOHA can simulate TWTs with non constant helix pitch or radius, which is impossible with the pseudospectral method used in RUBEUS. All these feats, coupled with the stability of its symplectic time integrator, enable DIMOHA to simulate long time steady states, supporting defects and reflected backward waves.

Finally, we validate DIMOHA for a continuous broadband impulse in both small and large signal regimes, comparing DIMOHA simulations of a severed helix TWT to multiple measurements and competing algorithms.

With a reasonable time cost, a good stability and the ability to simulate complex multi-frequency signal amplification,
DIMOHA can be used for real telecommunication signals processing, as well as defects and multiple reflections simulation.

\section{ACKNOWLEDGEMENTS}

We thank A. Poyé and B. Husquinet for fruitful and thoughtful discussions, and J. Noble for her comments on the manuscript. The Centre de Calcul Intensif d'Aix-Marseille is acknowledged for granting access to its high-performance computing resources.

\section{REFERENCES}

[1] D. Safi, P. Birtel, S. Meyne, and A. F. Jacob, "A traveling-wave tube simulation approach with cst particle studio," IEEE Transactions on Electron Devices, vol. 65, no. 6, pp. 2257-2263, 2018.

[2] J. Wöhlbier, M. Converse, J. Plouin, A. Rawal, A. Singh, and J. Booske, "LATTE/MUSE numerical suite: an open source teaching and research code for traveling wave tube amplifiers," in 4th IEEE International Conference on Vacuum Electronics, 2003, Apr. 2003, pp. 16-17. [Online]. Available: 10.1109/IVEC.2003.1286003

[3] S. Datta, P. Jain, M. Narayan, and B. Basu, "Nonlinear eulerian hydrodynamical analysis of helix traveling-wave tubes," IEEE Transactions on Electron Devices, vol. 45, no. 9, pp. 2055-2062, sep. 1998. [Online]. Available: 10.1109/16.711374

[4] D. F. G. Minenna, Y. Elskens, F. André, A. Poyé, J. Puech, and F. Doveil, "DIMOHA: A time-domain algorithm for traveling-wave tube simulations," IEEE Transactions on Electron Devices, vol. 66, no. 9, pp. 4042-4047, Jul. 2019. [Online]. Available: 10.1109/TED.2019.2928450

[5] D. F. G. Minenna, K. Aliane, Y. Elskens, A. Poyé, F. André, J. Puech, and F. Doveil, "Time simulation of the nonlinear wave-particle interaction in meters-long traveling-wave tubes," Physics of Plasmas, vol. 28, no. 9, p. 092110, 2021. [Online]. Available: https://doi.org/10.1063/5.0059349

[6] M. Converse, J. Booske, and S. Hagness, "Impulse amplification in a traveling-wave tube-I: Simulation and experimental validation," IEEE Transactions on Plasma Science, vol. 32, no. 3, pp. 1040-1048, Jun 2004. [Online]. Available: 10.1109/TPS.2004.828798

[7] M. Converse and J. Booske, "Impulse amplification in a traveling-wave tube-II: Large signal physics," IEEE Transactions on Plasma Science, vol. 32, no. 3, pp. 1049-1056, Jun. 2004. [Online]. Available: 10.1109/TPS.2004.828788

[8] A. Setayesh and M. S. Abrishamian, "PaWaIC-PSAOFDTD: Particlewave interaction code with pseudospectral arbitrary-order accurate temporal and spatial derivatives FDTD technique for helix TWT," IEEE Transactions on Electron Devices, vol. 64, no. 11, pp. 4706-4714, Nov. 2017. [Online]. Available: 10.1109/TED.2017.2757279

[9] M. Converse, "Investigation of the mechanisms of pulse amplification in helical traveling wave tubes," Ph.D. dissertation, University of Wisconsin-Madison, 2003.

[10] Q. H. Liu, "The PSTD algorithm: A time-domain method requiring only two cells per wavelength," Microwave and Optical Technology Letters, vol. 15, no. 3, pp. 158-165, Dec. 1997. [Online]. Available: 10. 1002/(SICI) 1098-2760(19970620)15:3〈158::AID-MOP11〉3.0.CO;2-3

[11] F. André, P. Bernardi, N. M. Ryskin, F. Doveil, and Y. Elskens, "Hamiltonian description of self-consistent wave-particle dynamics in a periodic structure," EPL (Europhysics Letters), vol. 103, no. 2, p. 28004, Jul. 2013. [Online]. Available: 10.1209/0295-5075/103/28004

[12] S. Ramo, "Currents induced by electron motion," Proceedings of the IRE, vol. 27, no. 9, pp. 584-585, Sep. 1939. [Online]. Available: 10.1109/JRPROC.1939.228757

[13] F. André, D. F. G. Minenna, K. Aliane, Y. Elskens, A. Poyé, and F. Doveil, "Progress with DIMOHA for fast time-domain simulations of traveling-wave tube," in 21st Int. Vac. Electron. Conf. (IVEC), Online, 2020.

[14] P. Waller, "Modélisation numérique de l'interaction et diagnostic expérimental du faisceau d'électrons dans un tube à ondes progressives spatial," Ph.D. dissertation, Univ. Paris Diderot, 1999.

[15] A. Singh, "Experimental investigation of TWT nonlinearities and distortion suppression by signal injection," Master's thesis, University of Wisconsin-Madison, 2003. 